\title{
Reply to 'Comment on 'Nomogram to predict pathologic complete response in HER2-positive breast cancer treated with neoadjuvant systemic therapy"
}

\author{
Takeo Fujii ${ }^{1,2}$ and Naoto T Ueno*,1
}

${ }^{1}$ Department of Breast Medical Oncology, Section of Translational Breast Cancer Research, The University of Texas MD Anderson Cancer Center, 1515 Holcombe Boulevard, Houston, TX 77030, USA and ${ }^{2}$ Department of Biostatistics, The University of Texas Health Science Center at Houston School of Public Health, 1200 Pressler Street, Houston, TX 77030, USA

Sir,

We thank K Altundag for the interest in our article (Fujii et al, 2017) and thoughtful comments. Kurozumi et al (2015) reported that Ki67 level and histological grade were significantly associated with pathological complete response (pCR) after neoadjuvant chemotherapy with trastuzumab in patients with HER2-positive breast cancer.

Polley et al (2013) reported that interlaboratory reproducibility of Ki67 was moderate. Although central staining demonstrated a better intraclass correlation coefficient: 0.71 (95\% confidence interval, $0.47-0.78$ ), in the setting of local staining, the intraclass correlation coefficient was low: 0.59 (95\% confidence interval, $0.37-0.68$ ). Also, the Ki67 value in patients who undergo neoadjuvant chemotherapy without endocrine therapy is still not obvious (Dowsett et al, 2011), and the National Comprehensive Cancer Network Clinical Practice Guidelines in Oncology (https:// www.nccn.org/professionals/physician_gls/f_guidelines.asp) do not recommend testing Ki67 routinely. Because of its low reproducibility and lack of recommendation as a standard of care, Ki67 staining is not routinely performed as standard practice. Therefore, we excluded Ki67 from our analysis to make our nomogram practical.

For histological grade, the hazard ratio was not significantly associated with pCR in multivariate analysis, despite the histological grade being a significant variable in univariate analysis in the study by Kurozumi et al (2015). Therefore, we assume that the effect of not including nuclear or histological grade in our analysis was minimal.

On the basis of these previous publications, many patients in our data set did not have Ki67 and histological grade. We agree with you that we cannot conclude anything about the clinical importance of Ki67 and histological grade in the context of neoadjuvant chemotherapy in patients with HER2-positive primary breast cancer unless we assess them in our model because this is a retrospective study.

In conclusion, the effect of not including Ki67 and histological grade in our analysis is small, but we cannot make a definite conclusion unless we perform the same analysis including Ki67 and histological grade.

\section{CONFLICT OF INTEREST}

The authors declare no conflict of interest.

\section{REFERENCES}

Dowsett M, Nielsen TO, A'hern R, Bartlett J, Coombes RC, Cuzick J, Ellis M, Henry NL, Hugh JC, Lively T, Mcshane L, Paik S, Penault-Llorca F, Prudkin L, Regan M, Salter J, Sotiriou C, Smith IE, Viale G, Zujewski JA, Hayes DF (2011) Assessment of Ki67 in breast cancer: recommendations from the International Ki67 in Breast Cancer Working Group. J Natl Cancer Inst 103: 1656-1664.

Fujii T, Kogawa T, Wu J, Sahin AA, Liu DD, Chavez-Macgregor M, Giordano SH, Raghavendra A, Murthy RK, Tripathy D, Shen Y, Yamal JM, Ueno NT (2017) Nomogram to predict pathologic complete response in HER2-positive breast cancer treated with neoadjuvant systemic therapy. Br J Cancer 116: 509-514.

Kurozumi S, Inoue K, Takei H, Matsumoto H, Kurosumi M, Horiguchi J, Takeyoshi I, Oyama T (2015) ER, PgR, Ki67, p27(Kip1), and histological grade as predictors of pathological complete response in patients with HER2-positive breast cancer receiving neoadjuvant chemotherapy using taxanes followed by fluorouracil, epirubicin, and cyclophosphamide concomitant with trastuzumab. $B M C$ Cancer 15: 622 .

Polley M-YC, Leung SCY, Mcshane LM, Gao D, Hugh JC, Mastropasqua MG, Viale G, Zabaglo LA, Penault-Llorca F, Bartlett JMS, Gown AM, Symmans WF, Piper T, Mehl E, Enos RA, Hayes DF, Dowsett M, Nielsen TO (2013) An International Ki67 Reproducibility Study. J Natl Cancer Inst 105: 1897-1906.

This work is published under the BJC's standard license to publish agreement. After 12 months the license terms will change to a Creative Commons AttributionNonCommercial-Share Alike 4.0 Unported License. 